\title{
Dipeptidyl peptidase I activates neutrophil-derived serine proteases and regulates the development of acute experimental arthritis
}

\author{
April M. Adkison, ${ }^{1}$ Sofia Z. Raptis, ${ }^{1}$ Diane G. Kelley, ${ }^{2}$ and Christine T.N. Pham ${ }^{1,3}$ \\ ${ }^{1}$ Department of Internal Medicine, \\ ${ }^{2}$ Department of Pediatrics, and \\ ${ }^{3}$ Department of Pathology and Immunology, Washington University School of Medicine, St. Louis, Missouri, USA
}

Address correspondence to: Christine T.N. Pham, 660 South Euclid Avenue, Campus Box 8045, St. Louis, Missouri 63110-1093. Phone: (314) 362-9043; Fax: (314) 454-1091; E-mail: cpham@im.wustl.edu.

Received for publication June 7, 2001, and accepted in revised form December 19, 2001.

Leukocyte recruitment in inflammation is critical for host defense, but excessive accumulation of inflammatory cells can lead to tissue damage. Neutrophil-derived serine proteases (cathepsin G [CG], neutrophil elastase [NE], and proteinase 3 [PR3]) are expressed specifically in mature neutrophils and are thought to play an important role in inflammation. To investigate the role of these proteases in inflammation, we generated a mouse deficient in dipeptidyl peptidase I (DPPI) and established that DPPI is required for the full activation of CG, NE, and PR3. Although $D P P I^{-/-}$mice have normal in vitro neutrophil chemotaxis and in vivo neutrophil accumulation during sterile peritonitis, they are protected against acute arthritis induced by passive transfer of monoclonal antibodies against type II collagen. Specifically, there is no accumulation of neutrophils in the joints of $\mathrm{PPPI}^{-/-}$mice. This protective effect correlates with the inactivation of neutrophil-derived serine proteases, since $\mathrm{NE}^{-/-} \times \mathrm{CG}^{-/-}$ mice are equally resistant to arthritis induction by anti-collagen antibodies. In addition, protease-deficient mice have decreased response to zymosan- and immune complex-mediated inflammation in the subcutaneous air pouch. This defect is accompanied by a decrease in local production of TNF- $\alpha$ and IL-1 $\beta$. These results implicate DPPI and polymorphonuclear neutrophil-derived serine proteases in the regulation of cytokine production at sites of inflammation.

J. Clin. Invest. 109:363-371 (2002). DOI:10.1172/JCI200213462.

\section{Introduction}

Polymorphonuclear neutrophils (PMNs) play a critical role in host defense against bacterial pathogens. PMNs elaborate a number of proteases that are involved in host immunity and are capable of bactericidal activity $(1,2)$. However, proteolytic enzymes released by PMNs also degrade extracellular matrix components, leading to tissue damage and chronic inflammation $(3,4)$. For example, PMNs are a prominent feature of joint inflammation in rheumatoid arthritis, and are thought to be responsible for the cartilage destruction in this disease. Therefore, the prevention of PMN migration and accumulation at inflammatory sites may limit their host-damaging effector functions.

PMNs express a family of neutral serine proteases in their azurophil (primary) granules. These PMN-specific, granule-associated enzymes include cathepsin G (CG), neutrophil elastase (NE), and proteinase 3 (PR3) (5). Another structurally related granule-associated protein is azurocidin, which has no enzymatic activity (6). While their protective role in host immunity is acknowledged $(7,8)$, the role of PMN-derived serine proteases in neutrophil extravasation at sites of inflammation is still controversial.

Due to their proteolytic properties, it has long been commonly thought that the main function of these neutrophil-derived proteases at sites of inflammation is to degrade various components of the extracellular matrix. The role of serine proteases in PMN recruitment, however, appears to be more complex than simple disruption of extracellular matrix. Recent evidence suggests that extracellularly released serine proteases remain catalytically active (9), and may modulate neutrophil recruitment. Inhibition of PMN-derived serine proteases has been shown to reduce PMN adhesion to various extracellular matrix-coated surfaces and endothelial layers ex vivo $(10,11)$, and neutrophil infiltration into inflamed tissues in vivo $(12,13)$. However, studies using naturally occurring protease inhibitors, as well as low-molecular-weight inhibitors, have shown that serine proteases are not required for successful PMN transendothelial migration in vitro (14), nor are they involved in the disruption of subendothelial basement membrane during PMN diapedesis (15).

Recently, proteolysis has emerged as an important mechanism of regulating cytokine bioactivity (16). For example, IL- $1 \beta$ and TNF- $\alpha$ are proteolytically processed to their active form by IL- $1 \beta$-converting enzyme (ICE, or caspase- 1 ) and TNF- $\alpha$-converting enzyme (TACE), respectively $(17,18)$. At sites of inflammation, elevated levels of extracellularly released, neutrophil-derived serine proteases temporally coincide with high concentra- 
tions of bioactive cytokines. This led to the hypothesis that PMN-derived serine proteases may control inflammatory processes through the proteolytic modification and release of cytokines. However, the extent of their regulatory functions in various pathophysiological conditions remains largely uncharacterized.

Serine proteases share a high degree of homology, both at the cDNA and protein levels. Genetic analysis shows that all of these serine proteases are synthesized as preproenzymes with a residue leader sequence of 18-26 amino acids that is cleaved, leaving a prodipeptide between the proenzyme and the mature active enzyme (19). The activation of these proteases requires the removal of this prodipeptide (20). We recently generated a mouse with a null mutation in the lysosomal cysteine protease dipeptidyl peptidase I (DPPI), and showed that DPPI is essential for the processing and activation of many serine proteases, including cytotoxic lymphocyte granzymes A and B (21) and mast cell chymase (22).

In this study, we established that DPPI is important for the processing and activation of the PMN-derived serine proteases NE, CG, and PR3. Although DPPI- and serine protease-deficient mice have normal in vitro chemotaxis and in vivo peritonitis in response to thioglycollate, they are resistant to acute arthritis induction by passive transfer of anti-type II collagen antibodies. Moreover, they have a defect in neutrophil recruitment in subcutaneous air pouches in response to zymosan and immune complexes. The decrease in inflammatory cell accumulation correlates with a decrease in cytokine production. These studies support the hypothesis that neutrophil-derived serine proteases are involved in the regulation of cytokine production at sites of inflammation.

\section{Methods}

Animals. DPPI ${ }^{-/-}, \mathrm{CG}^{-/-}, \mathrm{NE}^{-/-}$, and $\mathrm{NE}^{-/-} \times \mathrm{CG}^{-/-}$mice, all in the 129 genetic background, were produced as previously described $(7,21,23)$. These mice and wildtype (WT) control mice were kept in pathogen-free conditions at Washington University Specialized Research Facility. For in vitro analysis, mice $4-8$ weeks of age were used. For in vivo experiments, male mice 6-8 weeks of age were used.

Western blot analysis. Protein lysates were prepared from PMNs or bone marrow as previously described (23). Equivalent amounts of total protein were loaded onto $10 \%$ SDS-PAGE gels, transferred to nitrocellulose, and blotted with anti-NE and anti-CG antisera as previously described $(7,23)$. $\beta$-actin served as control for quantity and quality of protein.

S1 analysis. Total cellular RNA was prepared from bone marrow cells and analyzed by $\mathrm{S} 1$ nuclease protection assay using CG-specific probe as previously described (23). Murine $\beta_{2}$ microglobulin served as control for quantity and quality of RNA.

Determination of enzymatic activity. Total bone marrow cell lysates were prepared as previously described (23) and normalized for protein content. NE activity was measured using the peptide substrate $\mathrm{N}$-methoxysuc-
cinyl-Ala-Ala-Pro-Val-pNA (Elastin Products Co., Owensville, Missouri, USA); CG activity was measured using the substrate N-succinyl-Ala-Ala-Pro-Phe-pNA (Sigma-Aldrich, St. Louis, Missouri, USA); and PR3 activity was measured using the substrate $\mathrm{N}$-t-butyloxycarbonyl-Ala-Ala-Nva-thiobenzyl ester (Elastin Products Co.), as previously described (23).

In vitro chemotaxis. Neutrophils were purified from bone marrow using a discontinuous Percoll gradient (Amersham Pharmacia Biotech, Piscataway, New Jersey, USA). Neutrophil purity was consistently $75-85 \%$, as assessed by light microscopy of Wright-Giemsa-stained cytospins. Neutrophils were labeled with calcein AM (Molecular Probes Inc., Eugene, Oregon, USA), and placed on top of a 96-well chemotaxis chamber (Neuro Probe Inc., Gaithersburg, Maryland, USA). The bottom wells of the microplate were filled with either the chemotactic agent fMLP $\left(10^{-4} \mathrm{M}\right)$, or zymosan-activated rat serum $(2 \%$, as a source of $\mathrm{C} 5 \mathrm{a})$, or recombinant human IL-8 (rhIL-8) (3 $\mu \mathrm{g} / \mathrm{ml}$; Amersham Pharmacia Biotech), or buffer. After incubation for 1 hour at $37^{\circ} \mathrm{C}$ in an atmosphere of $5 \% \mathrm{CO}_{2}$, the nonmigrating cells from the top of the filter were removed, and the cells (tested in triplicate per point) that migrated to the bottom chamber were measured using a fluorescent plate reader (Bio-Tek Instruments Inc., Winooski, Vermont, USA). The fluorescence for each well was expressed as a percentage of total PMNs.

Superoxide production. Purified bone marrow-derived neutrophils were resuspended in HBSS containing $1.3 \mathrm{mM} \mathrm{CaCl}_{2}$ and $0.4 \mathrm{mM} \mathrm{MgSO}_{4}$, and stimulated with PMA in the presence of $0.2 \mathrm{mM}$ cytochrome $c$. The kinetics of superoxide dismutase-inhibitable reduction of cytochrome $c$ was assessed by measuring nmol of $\mathrm{O}_{2}-$ produced by $10^{6} \mathrm{PMNs}$ over a period of 30 minutes, at $\mathrm{OD}_{550}$.

Thioglycollate-induced peritonitis. Mice were injected with $1 \mathrm{ml}$ of thioglycollate broth intraperitoneally, and at the indicated timepoints, peritoneal cells were harvested by lavage and quantified.

Artbritisinduced by anti-type II collagen antibody. Mice were injected intravenously with a cocktail of $4 \mathrm{mg}$ of anti-type II collagen antibodies (Chemicon International Inc., Temecula, California, USA) on day 0 , followed by $50 \mu \mathrm{g}$ of intraperitoneal LPS on day 3 as recommended by the manufacturer. Severity of arthritis in each paw was scored as follows: $0=$ normal; $1=$ mild redness and swelling of digits, ankle, or wrist; 2 = moderate swelling of ankle or wrist; 3 = severe swelling involving entire paw, including digits (maximum score of 12 per mouse).

Histological grading of arthritis. At least four mice per genotype were sacrificed, and all four paws of each mouse were excised and fixed in $10 \%$ buffered formalin. Fixed tissues were decalcified in 14\% EDTA for 2 weeks, and then dehydrated and embedded in paraffin. Sagittal sections of talocrural or radiocarpal joints were stained with either hematoxylin and eosin or toluidine blue using standard techniques. Cells infiltrating the synovial and subsynovial space were identified by morphology 
and photographed using the LE-Digital 1-CCD video camera system (Optronics, Goleta, California, USA). Cell counts and differentials of four random high-power fields (HPFs) of each joint section were performed by the same blinded observer. Joint exudates were scored on a scale of 0 (normal) to 5 (severe, with presence of inflammatory cells fibrin-like material occupying more than $60 \%$ of the joint space). Proteoglycan depletion was scored on toluidine blue-stained sections on a scale of $0-3(0=$ fully stained cartilage, $1=$ less than $25 \%$ unstained cartilage, $2=25-50 \%$ unstained cartilage, and $3=$ more than $50 \%$ unstained cartilage).

Subcutaneous air pouch. The air pouches were formed according to the method of Edwards et al. (24). Mice were anesthetized, and air pouches were created by subcutaneous injection of $5 \mathrm{ml}$ of sterile air into the back. The pouches were reinflated 3 days later with $3 \mathrm{ml}$ of sterile air. On day 6 , the pouches were instilled with either $1 \mu \mathrm{g}$ of rIL-8, $0.3 \mathrm{ml}$ of $1 \%$ zymosan, or normal saline. At the indicated times, the mice were sacrificed, the pouches were lavaged with $5 \mathrm{ml}$ of PBS, and the number of PMNs was determined.

Reverse passive Arthus reaction. Mice were injected intravenously with $20 \mathrm{mg} / \mathrm{kg}$ chicken ovalbumin (OVA), followed by instillation of $300 \mu \mathrm{g}$ heat-inactivated rabbit anti-OVA antibodies (Sigma-Aldrich) in the air pouch to induce immune complex formation in situ. Mice treated with normal saline in the air pouch after intravenous injection of OVA served as controls. At indicated times after challenge, mice were sacrificed, and their pouches were lavaged as above.

Induction and measurement of cytokines. Mice were injected intraperitoneally with $50 \mu \mathrm{g}$ or $100 \mu \mathrm{g}$ of E. coli LPS, serotype 0127:B8 (Sigma-Aldrich), in $100 \mu \mathrm{l}$ of sterile PBS. Sera were collected before and 90 minutes after LPS challenge. TNF- $\alpha$ levels were determined using an ELISA kit (R\&D Systems Inc., Minneapolis, Minnesota, USA) per the manufacturer's protocol. TNF- $\alpha$ and IL- $1 \beta$ levels in air pouch supernatants collected at 12 hours after injection were also measured by ELISA as recommended by the manufacturer.
In vitro neutrophil degranulation. In vitro degranulation assays were performed as previously described (25). Bone marrow-derived neutrophils were resuspended in HBSS at $10^{7} / \mathrm{ml}$, and stimulated with $50 \mathrm{ng} / \mathrm{ml}$ PMA for 15 minutes. The cells were pelleted, and the supernatant was analyzed by gelatin zymography for matrix metalloproteinase 9 (MMP-9) activity. Briefly, the supernatant was subjected to SDS-PAGE on gels containing $1 \%$ gelatin under nonreducing conditions. After electrophoresis, gels were washed twice with $2.5 \%$ Triton X-100 for 30 minutes to remove SDS, and then incubated overnight at $37^{\circ} \mathrm{C}$ in reaction buffer containing $50 \mathrm{mM}$ Tris ( $\mathrm{pH} 7.4$ ), $150 \mathrm{mM} \mathrm{NaCl}$, and $5 \mathrm{mM}$ $\mathrm{CaCl}_{2}$. Gelatinolytic activity appeared as colorless bands against a blue background when stained with 0.125\% Coomassie brilliant blue.

Statistical analysis. Wilcoxon's two-tailed rank sum test for unpaired variables was used to compare differences between groups. $P$ values of less than 0.05 were considered significant.

\section{Results}

DPPI-/- mice have normal granulocyte development. We analyzed the hematopoietic development of multiple $D P^{-1-}$ mice derived from two different embryonic stem cell lines. Complete blood counts and differentials showed normal counts of circulating white blood cells (WBCs) and mature granulocytes (PMNs) in both WT $\left(9.08 \pm 2.7 \times 10^{3} \mathrm{WBCs} / \mu \mathrm{l}\right.$ with $24.7 \% \pm 7.7 \%$ $\mathrm{PMNs})$ and $D \mathrm{PPI}^{-/}$animals $\left(8.79 \pm 3.48 \times 10^{3}\right.$ WBCs $/ \mu 1$ with $24.4 \% \pm 10.9 \%$ PMNs). Cytospins of bone marrow cells derived from 4- to 8-week-old WT and $\mathrm{DPPI}^{-/-}$mice revealed normal numbers of myeloid precursors and mature granulocytes (data not shown). Transmission electron microscopy of bone marrow-derived PMNs from $D P P I^{-/-}$mice showed normal numbers of electron-dense azurophil granules and normal neutrophil morphology (data not shown). In addition, we have previously shown that PMNs derived from $\mathrm{DPPI}^{-/-}$mice express normal surface levels of Gr-1 and Mac-1 (21).

\section{Figure 1}

Analysis of PMN-derived serine proteases in $\mathrm{DPPI}^{-/-}$mice. $\mathrm{NE}(\mathbf{a})$ and $\mathrm{CG}(\mathbf{b})$ protein expression in $\mathrm{DPPl}^{-/-}$bone marrow lysates. $\beta$-actin served as control for protein content. Note the markedly reduced level of immunoreactive CG in $\mathrm{DPPI}^{-/-}$bone marrow lysate compared with WT. (c) $\mathrm{S} 1$ nuclease protection assay revealed equivalent levels of CG mRNA in $\mathrm{DPPl}^{-/-}$and WT bone marrow lysate. $\beta_{2}$ microglobulin $\left(\beta_{2} \mathrm{~m}\right)$ served as control for RNA loading. (d) $\mathrm{DPPl}^{-/-}$bone marrow cells and $\mathrm{NE}^{-/-}$bone marrow cells have equivalent residual levels of NE activity. (e) Both $\mathrm{DPPI}^{--}$and $\mathrm{CG}^{-1-}$ bone marrow cells have no detectable hydrolysis of the CG-specific peptide substrate. (f) Conversion of the PR3 substrate by $\mathrm{DPPI}^{-/-}$ bone marrow cell lysate was reduced by $80 \%$ compared with WT. Relative enzyme activity was measured at $\mathrm{OD}_{405}$ per $10^{5}$ bone marrow cells and values represent mean activity \pm SEM of at least three animals.
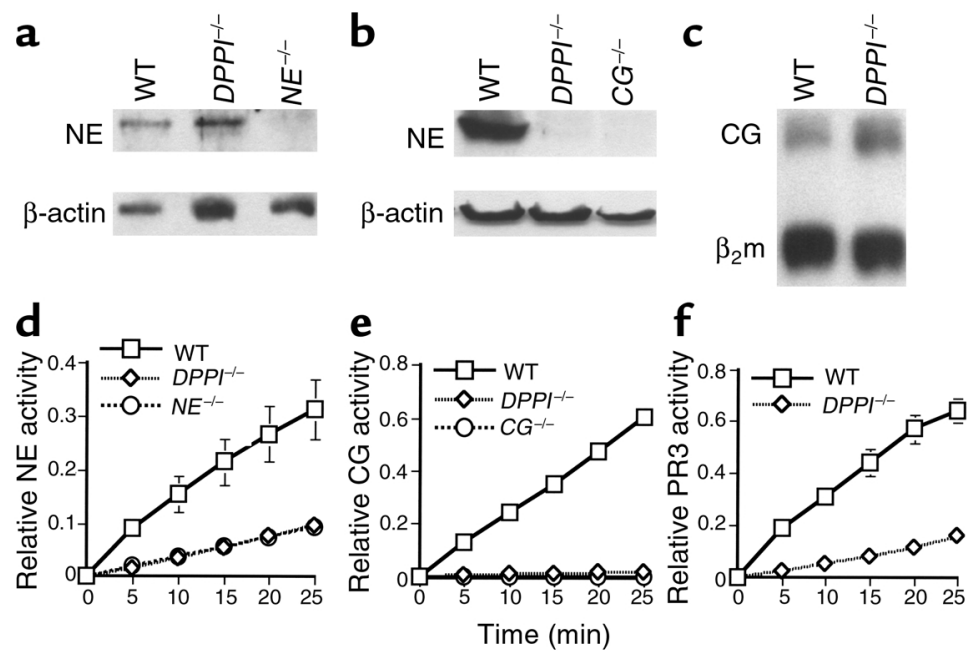

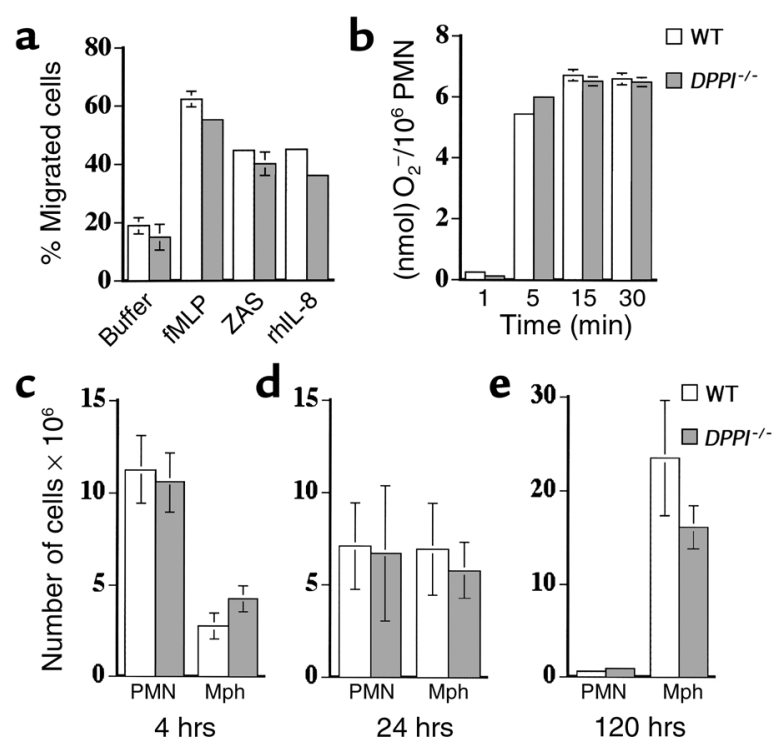

\section{Figure 2}

In vitro PMN function and in vivo response to thioglycollate in the absence of DPPI. (a) Bone marrow-derived PMNs from WT and $D \mathrm{PPI}^{-1-}$ mice were allowed to migrate across a filter in response to $10^{-4} \mathrm{M}$ fMLP, $2 \%$ zymosan-activated rat serum (ZAS), or $3 \mu \mathrm{g} / \mathrm{ml}$ rhIL-8. The number of cells that migrated was expressed as a percentage of total PMNs. Values represent the mean \pm SEM of at least three animals. (b) Superoxide production by PMNs in response to PMA, expressed as nmol of $\mathrm{O}_{2}^{-}$generated per $10^{6} \mathrm{PMNs}(n>3$ per genotype). The number of PMNs and macrophages recruited in response to intraperitoneal injection of thioglycollate is normal in $\mathrm{DPPI}^{-/-}$mice at 4 hours (c) 24 hours (d) and 120 hours (e). The slightly lower number of macrophages at 120 hours after thioglycollate injection in the $\mathrm{DPPI}^{-/-}$mice did not reach statistical significance ( $n=4-5$ mice per genotype). Mph, macrophages.

Serine protease activity is severely reduced in $\mathrm{DPPI}^{-/-}$neutrophils. Western blot analysis using NE-specific polyclonal rabbit anti-mouse sera confirmed equal immunoreactive NE bands in WT and $\mathrm{DPPI}^{-/-} \mathrm{PMN}$ lysates (Figure 1a). However, the amount of immunoreactive CG was markedly reduced in $D P P I^{-1-}$ lysates (Figure $1 \mathrm{~b}$ ), despite normal levels of mRNA detected by $\mathrm{S} 1$ analysis (Figure 1c). To rule out the possibility that this decreased level was due to nonspecific proteolytic activity that occurred after the cells were lysed, we performed immunohistochemistry on cytospin samples of bone marrow cells using the CG-specific antibody. We confirmed that immunoreactive levels of CG were reduced in $D P P I^{-/-}$PMNs prior to cell lysis (data not shown). Therefore, DPPI not only activates these proteases but may influence the total levels of these enzymes in some instances.

Using chromogenic substrates, we measured the specific activity of each granule-associated serine protease expressed in the myeloid compartment. NE activity in $D P P I^{-/-}$bone marrow lysate, as assessed by measuring the hydrolysis of $\mathrm{N}$-methoxysuccinyl-Ala-Ala-Pro-ValpNA, was equivalent to that seen in the $\mathrm{NE}^{-/-}$bone marrow extract (Figure 1d). CG activity, as assayed by the hydrolysis of N-succinyl-Ala-Ala-Pro-Phe-pNA, was vir- tually absent in $\mathrm{DPPI}^{-/-}$bone marrow lysate (Figure 1e). Lastly, PR3 activity, as assayed by the hydrolysis of N-tbutyloxycarbonyl-Ala-Ala-Nva-thiobenzyl ester, was reduced by $80 \%$ in $D P P I^{-/}$lysate (Figure 1f). It is difficult to assess whether this residual amount of activity is due to low levels of active PR3 enzyme, since PR3-deficient mice are currently unavailable. Since chromogenic substrates are not entirely specific for a given enzyme, it is likely that the apparent detection of residual NE and $\mathrm{PR} 3$ activities in $\mathrm{PPPI}^{-/}$- bone marrow is attributable to irrelevant proteases. However, we have previously shown that granzyme $\mathrm{C}$ and mast cell tryptase can be processed to the active form in the absence of DPPI $(21,22)$. Therefore, sensitive immunological assays that specifically detect the mature form of each of the three serine proteases will be necessary to firmly establish that $\mathrm{DPPI}^{-/}$ neutrophils lack all mature CG, NE, and PR3. Nevertheless, these results confirmed that DPPI plays an important role in the processing and full activation of all three PMN-derived serine proteases in vivo.

$D P P I^{-1-}$ mice have normal in vitro neutrophil chemotaxis and in vivo thioglycollate-induced peritonitis. Neutrophil chemotaxis was examined using a modified Boyden chamber. Calcein AM-labeled PMNs were allowed to migrate in response to fMLP $\left(10^{-4} \mathrm{M}\right)$, zymosan-activated rat serum $(2 \%)$, and rhIL-8 $(3 \mu \mathrm{g} / \mathrm{ml})$. There was no significant difference in the ability of $\mathrm{DPPI}^{-/} \mathrm{PMNs}$ to migrate toward these stimuli compared with that of WT PMNs (Figure 2a). In addition, $D P \mathrm{PI}^{-/-} \mathrm{PMNs}$ mobilized a normal respiratory burst in response to PMA, as determined by the kinetics of superoxide dismutase-inhibitable reduction of cytochrome $c$ (Figure $2 b$ ).

The response of $\mathrm{DPPI}^{-/-}$mice to thioglycollateinduced peritonitis was also assessed. Groups of 4-5 mice were injected intraperitoneally with $1 \mathrm{ml}$ of thioglycollate, and the peritoneal exudates were collected after 4 hours, 24 hours, and 120 hours. Thioglycollate injection into the peritoneum resulted in equivalent numbers of total leukocytes and PMNs recovered from WT mice and DPPI ${ }^{-/-}$mice at 4 hours and 24 hours (Figure 2, $\mathrm{c}$ and d). Peritoneal lavage at 120 hours after thioglycollate injection revealed no statistically significant difference between the numbers of WT and $\mathrm{DPPI}^{-/}$macrophages (Figure 2e). Thus, although the absence of DPPI severely reduced the activity of NE, CG, and PR3 in PMNs, the lack of serine protease activity did not affect in vitro neutrophil chemotaxis or in vivo recruitment of leukocytes in response to thioglycollate, a nonspecific inflammatory stimulus.

$D P I^{-/}$mice are resistant to induction of arthritis by passive transfer of anti-type II collagen antibodies. To further explore the role of DPPI and PMN-associated serine protease in a more pathophysiological condition, we challenged the DPPI-deficient mice to a well-established model of acute experimental arthritis (26). Four milligrams of a cocktail of four separate monoclonal antibodies specific for the main arthrogenic determinants of type II collagen were injected intravenously into groups of WT and $\mathrm{DPPI}^{-/-}$mice. On day 3, $50 \mu \mathrm{g}$ 
of LPS was injected intraperitoneally into each mouse. LPS, a strong inducer of proinflammatory cytokines, has been shown to act synergistically in the induction of arthritis (26). Joint inflammation developed within 24-48 hours after LPS injection, and peaked around day $6-7$. By day $7,87.5 \%$ of WT mice $(n=16)$ developed clinically evident arthritis, with a mean arthritis score of $6.3 \pm 3.3$ (maximum score, 12 per mouse). Arthritis is manifested by redness and swelling of the entire paw, including digits (Figure 3a). In contrast, none of the $D P P I^{--}$mice $(n=6)$ displayed any clinical signs of inflammation in either joints or digits (Figure 3b).

We performed histological analysis to more precisely determine the nature of the infiltrating cells. The front and hind paws of WT and $D P P I^{-/-}$mice were removed and processed. Hematoxylin and eosin-stained joint sections from WT mice revealed extensive infiltration of the subsynovial tissues by inflammatory cells (91 \pm 17 cells/HPF). PMNs were the predominant invading cells (78\%), with a smaller contribution from macrophages and lymphocytes (Figure 3, c, e, and g). In contrast, very few inflammatory cells $(9.1 \pm 2.2$ / HPF, $P<0.001)$ infiltrated the subsynovial space of $D P \mathrm{PI}^{-/-}$ joints (Figure 3, d, f, and h), and the majority of these cells had macrophage-like morphology. Inflammation was also assessed by the severity of the inflammatory exudate in the joint space, and by proteoglycan depletion on toluidine blue-stained sections. Abundant PMNs were also found in the joint space of WT mice, along with marked fibrin-like deposition (Figure 3, c and e). The inflammatory exudate adhered to the joint surface, which led to proteoglycan depletion, as evidenced by the loss of toluidine blue staining (Figure 3i). In contrast, $D P P I^{-/-}$joint sections showed no or very scant exudate (Figure 3, d and f), with preservation of proteoglycan content in cartilage (Figure $3 \mathrm{j}$ ). These results suggest that $D P \mathrm{PI}^{-/-}$joints were protected from $\mathrm{PMN}$ infiltration and cartilage destruction.

In this model of acute arthritis, cartilage-bound anti-type II collagen antibodies activate the classical pathway of the complement cascade, leading to the generation of activated complement components (27). Using immunohistochemistry, we confirmed that there were equivalent amounts of anti-type II collagen antibody and C3 deposition (and activation) on the cartilage surface of WT and $\mathrm{DPPI}^{-/-}$knee joints after intravenous injection of monoclonal antibodies (data not shown). To further confirm that the activation of the late complement components C5-C9 occurred normally in both genotypes, we performed hemolytic assays using sensitized sheep erythrocytes, and sera from WT and $\mathrm{DPPI}^{-/-}$mice. We found that serum from $D P P I^{-/-}$mice can activate the complement cascade and induce red blood cell lysis to the same extent as WT serum (data not shown). Therefore, the lack of inflammation in the $\mathrm{PPPI}^{-/-}$ mice is not due to a defect in antigen (type II collagen) recognition by the antibody, or a defect in the activation of the complement cascade.
To rule out the possibility that the absence of DPPI may adversely affect the release of granule-associated serine proteases, we performed in vitro degranulation experiments. Upon stimulation with $50 \mathrm{ng} / \mathrm{ml}$ of PMA, WT and $D P P I^{-/}$neutrophils released similar levels of MMP-9, as determined by gelatin zymography (Figure 4a). MMP-9 is one of the major proteases released by neutrophils upon activation, and is unaffected by the DPPI mutation. These results suggest that $\mathrm{DPPI}^{-/-}$neutrophils undergo normal degranulation with stimulation.

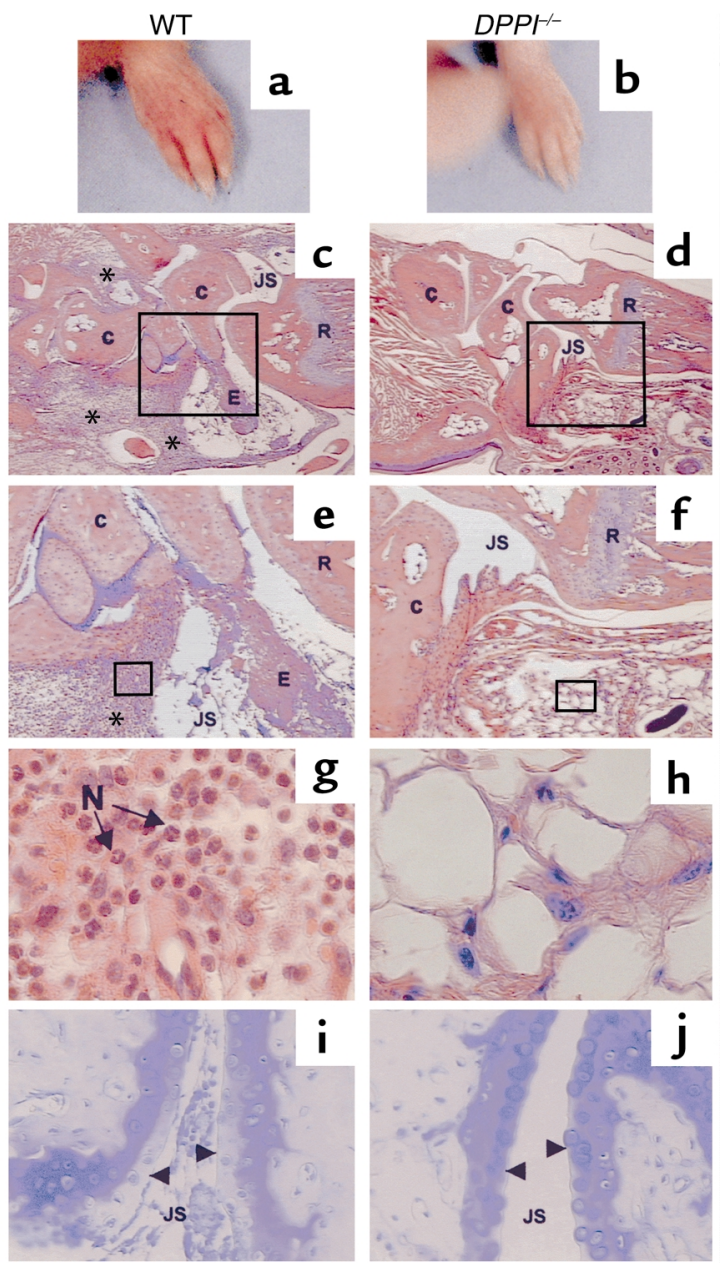

\section{Figure 3}

Clinical and histological assessment of monoclonal antibody-induced arthritis. Forepaw of a WT mouse (a) and a $\mathrm{PPPI}^{-/-}$mouse (b) on day 7 after receiving arthrogenic antibodies against type II collagen. Note the redness and swelling involving the entire paw, including the digits, of the WT mouse (a). Histological analysis of sections taken from the radiocarpal joints of immunized animals, stained with hematoxylin and eosin ( $\mathbf{c}-\mathbf{h}$ ), and toluidine blue (i and $\mathbf{j}$ ) (to detect proteoglycan). WT mice $(\mathbf{c}, \mathbf{e}, \mathbf{g}$, and $\mathbf{i})$ have marked leukocyte infiltration $\left({ }^{*}\right)$ in the subsynovial tissue and joint space, adherence of inflammatory cells to joint surfaces, and proteoglycan loss as evidenced by the reduction in metachromasia at the superficial border of the cartilage matrix (arrowheads). The infiltrating cells are predominantly neutrophils $(\mathrm{N})(\mathrm{g})$. $\mathrm{DPPl}^{-/-}$mice were protected from arthritis, as evidenced by normal joint histology, lack of inflammatory infiltrates, and preservation of proteoglycan content $(\mathbf{d}, \mathbf{f}, \mathbf{h}$, and $\mathbf{j})$. Magnification: $\mathbf{c}$ and $\mathbf{d}, \times 16$; $\mathbf{e}$ and $\mathbf{f}, \times 40$; $\mathbf{g}$ and $\mathbf{h}, \times 400$. E, exudate; $\mathrm{C}$, carpal bones; $\mathrm{R}$, radius; JS, joint space. 
a

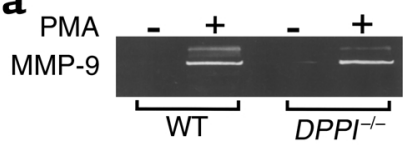

b

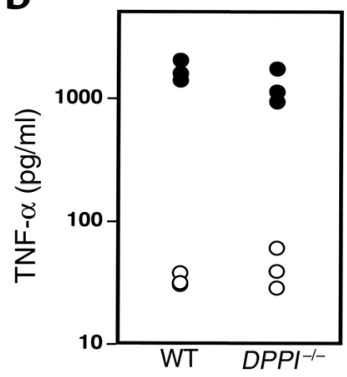

Figure 4

In vitro neutrophil degranulation and TNF- $\alpha$ production in $\mathrm{DPPI-/-}$ mice. (a) Purified PMNs $\left(10^{7} / \mathrm{ml}\right)$ were incubated with $50 \mathrm{ng} / \mathrm{ml}$ PMA for 15 minutes, and cell-free supernatants were analyzed by gelatin zymography. Note that $\mathrm{DPPI^{-1- }}$ and WT PMNs released equivalent levels of MMP-9. (b) TNF- $\alpha$ production 90 minutes after intraperitoneal injection of $100 \mu \mathrm{g}$ LPS. Open circles represent TNF- $\alpha$ levels prior to LPS injection. Serum TNF- $\alpha$ levels (filled circles) after LPS injection were equivalent in WT mice and $\mathrm{DPPI}{ }^{-/-}$mice.

In addition, a recent report suggests that mice deficient in both NE and CG are more resistant than WT mice are to the effects of LPS (8). Since TNF- $\alpha$ has been identified as the central cytokine released upon LPS stimulation, and plays a critical role in inflammatory joint disease (28), the possibility exists that reduced serum TNF- $\alpha$ levels in $D P \mathrm{PI}^{-/-}$mice upon LPS challenge is responsible for the resistance to arthritis. However, we found that 90 minutes after intraperitoneal injection of $100 \mu \mathrm{g}$ of LPS, WT and $\mathrm{DPPI}^{-/-}$mice had released similar levels of TNF- $\alpha$ into the serum (Figure $4 \mathrm{~b})$. We also performed the experiment using $50 \mu \mathrm{g}$ of LPS, with similar results (data not shown). Thus, the lack of inflammation in $\mathrm{DPPI}^{-/-}$mice is not due to a decrease in serum level of TNF- $\alpha$ in response to systemic administration of LPS.

Resistance to acute arthritis in DPPI ${ }^{-/}$mice correlates with the intrinsic defect in PMN-derived serine protease activity. $D P P I^{-/-}$mice have defects in many immune effector cell functions that may contribute to the observed phenotype. To further evaluate the mechanisms involved in the resistance of susceptibility, we tested whether the absence of NE and/or CG also conferred resistance to arthritis. Following injection with anti-type II collagen monoclonal antibodies and LPS, all $\mathrm{CG}^{-/-}$mice developed clinical arthritis, with a relatively low mean arthritic score $(3.3 \pm 1.5, P<0.1)$. NE deficiency alone considerably attenuated the phenotype; only $75 \%$ of $N E^{-/-}$mice developed clinically evident arthritis, with a mean score of $1.8 \pm 1.7(P<0.02)$. Similar to the $D P P I^{-/-}$ mice, the $N E^{-/-} \times C G^{-/-}$mice were all clinically resistant to the induction of arthritis (Figure 5a).

Histological analysis confirmed the scant presence of inflammatory cells in the subsynovial space of $N E^{-/-} \times \mathrm{CG}^{-/-}$mice $(5.8 \pm 2.2$ cells/HPF, $P<0.001)$ compared with WT mice $(91 \pm 17$ cells/HPF), little if any exudate in the joint space, and no proteoglycan loss (Figure 5, b-d). In accordance with the clinical data, $N E^{-/-}$mice and $C G^{-/-}$mice both had fewer

inflammatory cells infiltrating the subsynovial tissue space than did WT mice $\left(N E^{-/-}\right.$mice, $19 \pm 8.4$ cells/HPF, $P<0.001$; $C^{-/-}$mice, $42 \pm 29$ cells $/ \mathrm{HPF}$, $P<0.01$ ). In addition, both $N E^{-/-}$and $C G^{-/-}$joints showed less exudate and fibrin-like deposition in the joint space, along with some preservation of proteoglycan content in cartilage (Figure $5, \mathrm{c}$ and d). The fact that the single protease-deficient mice showed an intermediate phenotype indicates that both $\mathrm{NE}$ and CG contribute to the recruitment of inflammatory cells, and that the effect is additive.

$D P I^{-1}$ mice have decreased response to zymosan- and immune complex-induced inflammation in the subcutaneous air pouch. To further elucidate the mechanism(s) underlying the joint-protecting effect observed in the proteasedeficient mice, we used the air pouch model of inflammation. Air pouches formed by subcutaneous injection of air into the back of a mouse develop a lining layer resembling synovial membrane (24). The air pouch model therefore provides a suitable space to investigate the inflammatory responses in synovial-like tissues. Injection of zymosan into air pouches induced a neutrophil influx that peaked at 12 hours (29). This

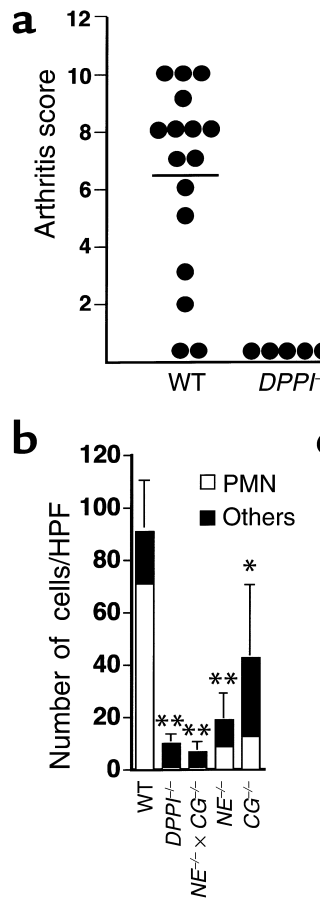

Figure 5

Summary of clinical arthritis and histological scores of immunized mice. (a) Groups of mice with different genotypes were immunized with arthrogenic antibodies on day 0 , followed by LPS on day 3 . Each paw was evaluated and scored on a scale of $0-3$, as outlined in Methods. Results are expressed as the mean arthritis score on day 7 of all four paws (maximum score of 12 per mouse). Joint sections were evaluated by a blinded observer for cellular infiltrate $(\mathbf{b})$, severity of joint exudate (c), and proteoglycan (PG) depletion(d). Degree of proteoglycan depletion was scored on a scale of $0-3$ with 3 being most severe. Results are expressed as mean \pm SEM for each of the histological features, quantified in WT, $D P P^{-1-}, N^{-/-} \times C^{-/-}, N E^{-/-}$, and $C^{-1-}$ mice $(n=$ at least four animals and 16 paws per genotype). ${ }^{*} P<0.01,{ }^{*} P<0.001$. 


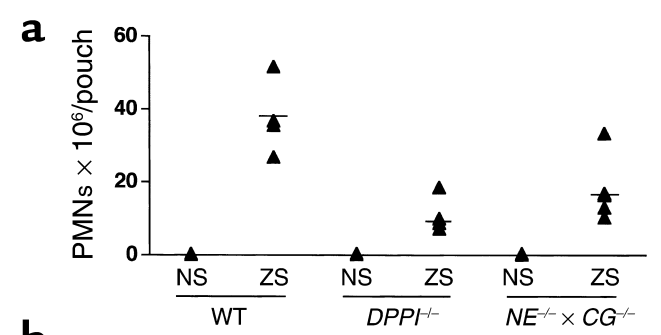

b
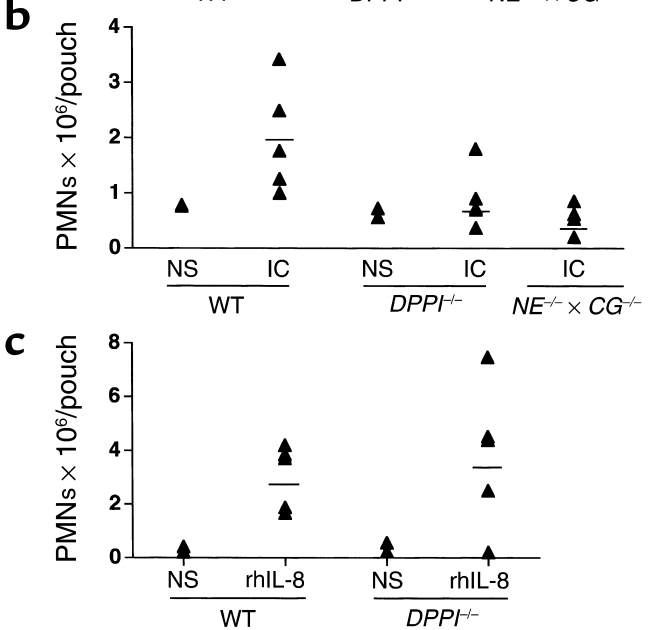

Figure 6

Neutrophil recruitment in air pouch model. Neutrophil recruitment in response to zymosan (ZS) (a), immune complex (IC) formation (b), and rhIL-8 (c). Mice were sacrificed 12 hours after injection of ZS and IC formation and 4 hours after rhIL-8. Each point represents the number of PMNs in an individual pouch. NS, normal saline.

response was reduced by $70 \%$ in the $\mathrm{DPPI}^{-/-}$mice $\left[(11.1 \pm 5.1) \times 10^{6} \mathrm{PMNs} /\right.$ pouch, $\left.n=4, P<0.01\right] \mathrm{com}-$ pared with WT mice $\left[(37.7 \pm 10.3) \times 10^{6} \mathrm{PMNs} /\right.$ pouch, $n=4]$ (Figure 6a). Neutrophil recruitment was also defective in the $\mathrm{NE}^{-/-} \times \mathrm{CG}^{-/-}$mice $\left[(18.0 \pm 9.0) \times 10^{6} \mathrm{PMNs} /\right.$ pouch, $n=5, P<0.02]$. A similar defect in neutrophil recruitment was seen with the reverse passive Arthus reaction. WT mice treated with both intravenous OVA and $300 \mu \mathrm{g}$ of anti-OVA in the air pouch to induce in situ formation of immune complexes developed a moderate but significant neutrophil accumulation in the pouches $\left[(2.0 \pm 0.9) \times 10^{6} \mathrm{PMNs} /\right.$ pouch, $\left.n=5\right]$ measured 12 hours after injection (Figure $6 \mathrm{~b}$ ). The number of emigrated PMNs was reduced in $D P P I^{-/}$mice $\left[(0.9 \pm 0.5) \times 10^{6}\right.$ PMNs/pouch, $n=5, P<0.1]$ and $N E^{-/-} \times C G^{-/-}$mice $\left[(0.6 \pm 0.3) \times 10^{6} \mathrm{PMNs} /\right.$ pouch, $\left.n=4, P<0.05\right]$ (Figure $6 \mathrm{~b})$. In fact, the response of $\mathrm{NE}^{-/-} \times \mathrm{CG}^{-/-}$mice to the treatment was equivalent to their response to injection of normal saline into air pouches $\left[(0.7 \pm 0.1) \times 10^{6}\right.$ PMNs/pouch, $n=4]$.

To investigate whether local cytokine production is normal in this model, we measured levels of TNF- $\alpha$ and IL-1 $\beta$ in the air pouches following zymosan injection. Although $\mathrm{DPPI} \mathrm{I}^{-/-}$mice and $\mathrm{NE}^{-/-} \times \mathrm{CG}^{-/-}$mice challenged systemically with LPS showed normal levels of TNF- $\alpha$ production in serum (ref. 8 and Figure $4 \mathrm{~b}$ ), we found that local production of TNF- $\alpha$ and IL- $1 \beta$ was decreased in air pouches of both $\mathrm{DPPI}^{-/-}$mice and
$\mathrm{NE}^{-/-} \times \mathrm{CG}^{-/-}$mice, compared with those of WT mice (Table 1). Injection of rhIL-8, a chemokine specific for neutrophils, into air pouches led to comparable levels of PMNs recruited in WT and DPPI-deficient mice $\left(3.0 \pm 1.2 \times 10^{6}\right.$ and $3.8 \pm 2.7 \times 10^{6} \mathrm{PMNs} /$ pouch, respectively, $n=5$ per genotype) (Figure 6c). Taken together, these results suggest that defective local production of cytokines (and probably chemokines) contributes to impaired neutrophil recruitment at sites of inflammation in $\mathrm{DPPI}^{-/-}$mice and $\mathrm{NE}^{-/-} \times \mathrm{CG}^{-/-}$mice.

\section{Discussion}

In this report, we establish that DPPI is essential for the full activation of CG, NE, and PR3 in vivo. Although cascades of proteolytic activation have been proposed and confirmed for several series of proteases, it is unlikely that DPPI initiates a sequence of events during which DPPI activates CG, which in turn activates NE, which then activates PR3. Using specific serine protease-deficient mice, we have previously shown that $\mathrm{CG}^{-/-}$neutrophils have a normal level of NE activity (23); similarly, $N E^{-/-}$neutrophils have normal CG activity (25). Since NE, CG, and PR3 activities are all severely reduced in $D P P I^{-/}$neutrophils, previous reports and this study suggest that all three serine proteases are substrates of DPPI. In addition, the activation of these proteases requires cleavage after a glutamic acid residue, a specificity that is different from the known specificity of each of these three serine proteases (30). We also found that $\mathrm{PPPI}^{-/-}$ PMNs contain significantly lower amounts of immunoreactive CG than are found in WT PMNs, but levels of steady-state mRNA that are basically equivalent to WT levels. This result is consistent with the previous observation that unprocessed granzyme $\mathrm{A}$ is more susceptible to degradation in the absence of DPPI (21). In addition, DPPI also influences the total amount of active tryptase in bone marrow-derived mast cells (22). Previous studies have shown that processing of neutrophilderived serine proteases is either part of the process of, or the result of, the sorting of these proteases to azurophil granules, where these enzymes are stored in their active form (31). However, sorting may also occur normally in the absence of the prodipeptide (32). Therefore, it is likely that in the absence of DPPI, these

\section{Table 1}

Defective cytokine production in $\mathrm{DPPI}^{-/-}$and $\mathrm{NE}^{-/-} \times \mathrm{CG}^{-/-}$mice

\begin{tabular}{lccc}
\hline Genotype & Treatment & $\begin{array}{c}\text { TNF- } \alpha \\
(\mathrm{pg} / \mathrm{ml})\end{array}$ & $\begin{array}{c}\mathrm{IL}-1 \beta \\
(\mathrm{pg} / \mathrm{ml})\end{array}$ \\
$\mathrm{WT}$ & Saline & $11 \pm 2$ & $9 \pm 1$ \\
& Zymosan & $60 \pm 9$ & $899 \pm 137$ \\
$\mathrm{DPPI} \mathrm{I}^{-/-}$ & Saline & $11 \pm 2$ & $33 \pm 9$ \\
& Zymosan & $35 \pm 7^{\mathrm{A}}$ & $538 \pm 144^{\mathrm{B}}$ \\
$\mathrm{NE}^{-/-} \times \mathrm{CG}^{-/-}$ & Saline & $11 \pm 1$ & $12 \pm 4$ \\
& Zymosan & $30 \pm 1^{\mathrm{C}}$ & $542 \pm 199^{\mathrm{B}}$
\end{tabular}

Groups of 4-5 mice were injected with $0.3 \mathrm{ml}$ of $1 \%$ zymosan or normal saline into air pouches created 6 days before. Animals were sacrificed 12 hours later, and the lavage fluid was assayed for the specified cytokine. ${ }^{A} P<0.01$, ${ }^{\mathrm{B}} P<0.02,{ }^{\mathrm{C}} P<0.001$. 
enzymes persist in a proform that can be missorted into a different granule compartment (32). Neutrophils express several classes of granules (azurophil, specific, gelatinase, and secretory), and missorting of azurophil granule serine proteases potentially targets them for degradation. The fact that the CG level is reduced while the NE level is not also suggests that individual granule proteases may not be sorted in an identical manner.

Although recent reports suggest that membranebound serine proteases play a role in chemotaxis $(33,34)$, we found that $\mathrm{DPPI}^{--}$PMNs migrate normally in vitro in response to optimal concentrations of various chemoattractants. In addition, using a model of nonspecific inflammation induced by intraperitoneal injection of thioglycollate, we showed that leukocyte recruitment into the peritoneum is normal in these mutant mice. These observations are in agreement with recent studies that report normal neutrophil chemotaxis and neutrophil accumulation during sterile and infectious peritonitis in $C G^{-/}, N E^{-/-}$, and $N E^{-/-} \times C G^{-/-}$mice $(7,8,23)$.

Recent evidence suggests that PMN-derived serine proteases are also expressed on the neutrophil surface, and are active and protected from naturally circulating inhibitors (9). This concentrated expression of serine proteases on the surface of PMNs could be important for the processing of cytokines. Several studies suggest that PMN-derived serine proteases are involved in the control of cytokine production. PR3 has been shown to be a potent activator of membrane-bound TNF- $\alpha$ and pro-IL-1 $\beta$ (35). CG, NE, and PR3 have been reported to catalyze the release of more active forms of CXC chemokines, such as IL-8 and epithelial cell-derived neutrophil activating protein-78 (ENA-78), through proteolytic processes $(36,37)$. In addition, PR3 and NE also enhance the production of IL-8 by endothelial cells $(38,39)$. Therefore, it is reasonable to hypothesize that the interaction between serine proteases and cytokines amplifies the necessary signals that sustain neutrophil recruitment at sites of inflammation.

Using a well-defined acute experimental arthritis model and the subcutaneous air pouch, we showed that the absence of DPPI and active serine proteases totally abrogated antibody-mediated acute arthritis, and significantly attenuated the response to zymosan and immune complex-induced inflammation in the subcutaneous air pouch. In the arthritis model, immune complex formation led to the activation of the classical pathway of the complement cascade and the generation of C5a. Although the activated complement component C5a is a potent chemoattractant, it has a short half-life. Other cytokines and factors may be needed for maximal inflammatory cell recruitment. NE has previously been shown to play a critical role in sustaining recruitment of PMNs in bullous pemphigoid induced in the mouse, another model of antibody-mediated inflammation of the skin (25). In that report, the authors hypothesize that NEmediated extracellular matrix degradation products, such as elastin fragments, are chemotactic for neutrophils. Our present studies extend the role of PMN-derived ser- ine proteases to regulatory functions in the cytokine network. Although $\mathrm{DPPI}^{-/-}$mice and $\mathrm{NE}^{-/-} \times \mathrm{CG}^{-/-}$mice produced normal serum levels of TNF- $\alpha$ in response to systemically administered LPS (ref. 8 and Figure 4b), the local cytokine response in the air pouch was reduced by approximately $50 \%$ compared with the response in WT mice. In addition, injection of IL-8, a chemokine specific for neutrophils, led to an equivalent number of PMNs recruited into DPPI-deficient air pouches. Taken together, these results support the concept that DPPI- and serine protease-deficient PMNs do not have an intrinsic defect in their ability to transmigrate across the endothelial barrier into the air pouch environment in response to CXC-like chemokines. Instead, the findings are in keeping with the concept that serine proteases modulate the local release and/or processing of cytokines, and possibly of chemokines. However, the possibility remains that $D P I^{-/-} \mathrm{PMNs}$ and $\mathrm{NE}^{-/-} \times \mathrm{CG}^{-/-} \mathrm{PMNs}$ respond differentially to other chemoattractants that are generated locally in the inflamed joint.

Alternatively, PMNs may preferentially use proteases to infiltrate certain extracellular matrix-rich environments (such as the joints) during specific inflammatory responses. In the extravascular space, directional cell motility depends on both adhesion and subsequent detachment. The extracellular expression of proteases could represent a general mechanism used by various cell types to regulate locomotion. For example, the binding of urokinase-type plasminogen activator (uPA, a serine protease) to its receptor is an important system regulating cell migration and invasiveness in normal and pathological conditions (40). The matrix metalloprotease MMP-2 colocalizes with the surface integrin $\alpha_{v} \beta_{3}$ on angiogenic blood vessels, and has been shown to play an essential role in angiogenesis by promoting cell migration (41). Recent evidence suggests that PMN-derived serine proteases expressed on the neutrophil surface may associate with CD11b/CD18 (Mac-1), the major integrin on the PMN surface (42). This concentrated expression of serine proteases on the surface of cells could be important for the detachment and locomotion of PMNs, especially in an extracellular matrix-rich environment such as the synovium. It is likely that at specific inflammatory sites, excessive adhesion may actually interfere with efficient movement of neutrophils. Therefore, surfacebound serine proteases may promote the release of PMNs from their integrin-mediated adhesion by cleaving the tethering association between integrins and their ligands. In fact, purified human NE has been shown to cleave surface ICAM-1 and recombinant soluble ICAM-1 (43), and both NE and CG can cleave VCAM-1 (44).

In summary, this study has established that DPPI is the major enzyme activating neutrophil-derived serine proteases in vivo. The importance of these proteases in inflammatory processes was demonstrated by the dramatic protection against arthritis development in an in vivo model of acute inflammatory arthritis. These observations also extend the role of neutrophil-derived proteases in inflammation beyond degradation of 
extracellular matrix components, and strongly implicate these proteases in the control of cytokine production. This amplification process by $\mathrm{PMN}$-derived serine proteases on the cytokine network points to new directions in therapeutic interventions that may potentially limit the deleterious effects of excessive neutrophil influx at sites of inflammation. Lastly, it would be of interest to investigate whether these enzymes also play a role in other arthritis models, especially those with more chronic inflammation in which PMNs are not the predominant cell type, such as arthritis induced by active immunization with heterologous type II collagen.

\section{Acknowledgments}

The authors wish to thank Timothy Ley and Steve Shapiro for kindly providing the $\mathrm{CG}^{-/-}, \mathrm{NE}^{-/-}$, and $\mathrm{NE}^{-/-}$ $\times \mathrm{CG}^{-/-}$mice, and the specific antibodies for CG and NE. We also thank Timothy Ley, Steve Shapiro, and Hector Molina for their many helpful discussions and critiques of the manuscript, and Alec Cheng and Dan Link for their invaluable comments. We thank Dale Kobayashi, Ron McCarthy, and Jami Sarsany from the Shapiro laboratory for their excellent technical help and advice. This work was supported by NIH grant BL03774 (C.T.N. Pham) and a Biomedical Science Grant from the Arthritis Foundation (C.T.N. Pham).

1. Lehrer, R.I., and Ganz, T. 1990. Antimicrobial polypeptides of human neutrophils. Blood. 76:2169-2181.

2. Chertov, O., Yang, D., Howard, O.M., and Oppenheim, J.J. 2000. Leukocyte granule proteins mobilize innate host defenses and adaptive immune responses. Immunol. Rev. 177:68-78.

3. Weiss, S.J. 1989. Tissue destruction by neutrophils. N. Engl. J. Med. 320:365-376.

4. Lehr, H., and Arfors, K. 1994. Mechanisms of tissue damage by leukocytes. Curr. Opin. Hematol. 1:92-99.

5. Caughey, G.H. 1994. Serine proteinases of mast cell and leukocyte granules. A league of their own. Am. J. Respir. Crit. Care Med. 150:S138-S142.

6. Gabay, J.E., and Almeida, R.P. 1993. Antibiotic peptides and serine protease homologs in human polymorphonuclear leukocytes: defensins and azurocidin. Curr. Opin. Immunol. 5:97-102.

7. Belaaouaj, A., et al. 1998. Mice lacking neutrophil elastase reveal impaired host defense against gram negative bacterial sepsis. Nat. Med. 4:615-618.

8. Tkalcevic, J., et al. 2000. Impaired immunity and enhanced resistance to endotoxin in the absence of neutrophil elastase and cathepsin G. Immunity. 12:201-210.

9. Owen, C.A., Campbell, M.A., Sannes, P.L., Boukedes, S.S., and Campbell, E.J. 1995. Cell surface-bound elastase and cathepsin G on human neutrophils: a novel, non-oxidative mechanism by which neutrophils focus and preserve catalytic activity of serine proteases. J. Biol. Chem. 131:775-789.

10. Carney, D.F., Jagels, M.A., Hugli, T.E., Sands, H., and Rubin, H. 1998. Alpha1-proteinase inhibitor, anti-chymotrypsin, and a recombinant hybrid mutant of anti-chymotrypsin (LEX-032) modulate neutrophil adhesion interactions. J. Leukoc. Biol. 63:75-82.

11. Delyani,J.A., Murohara, T., and Lefer, A.M. 1996. Novel recombinant serpin, LEX-032, attenuates myocardial reperfusion injury in cats. Am. J. Physiol. 270:H881-H887.

12. Zimmerman, B.J., and Granger, D.N. 1990. Reperfusion-induced leukocyte infiltration: role of elastase. Am. J. Physiol. 259:H390-H394.

13. Woodman, R.C., Reinhardt, P.H., Kanwar, S., Johnston, F.L., and Kubes, P. 1993. Effects of human neutrophil elastase (HNE) on neutrophil function in vitro and in inflamed microvessels. Blood. 82:2188-2195.

14. Mackerel, A.J., Cottell, D.C., Russell, K.J., Fitzgerald, M.X., and O'Connor, C.M. 1999. Migration of neutrophils across human pulmonary endothelial cells is not blocked by matrix metalloproteinase or serine protease inhibitors. Am.J. Respir. Cell Mol. Biol. 20:1209-1219.

15. Huber, A.R., and Weiss, S.J. 1989. Disruption of subendothelial basement during neutrophil diapedesis in an in vitro construct of a blood vessel wall. J. Clin. Invest. 83:1122-1136.

16. Bank, U., and Ansorge, S. 2001. More than destructive: neutrophil-derived serine proteases in cytokine bioactivity control. J. Lenkoc. Biol. 69:197-206.

17. Fantuzzi, G., and Dinarello, C.A. 1999. Interleukin-18 and interleukin-1 beta: two cytokine substrates for ICE (caspase-1). J. Clin. Immunol. 19:1-11.

18. Cerretti, D.P. 1999. Characterization of the tumor necrosis factor alpha-converting enzyme, TACE/ADAM17. Biochem. Soc. Trans. 27:219-223.

19. Jenne, D.E., and Tschopp, J. 1988. Granzymes, a family of serine proteases released from granules of cytolytic $T$ lymphocytes upon $T$ cell receptor stimulation. Immunol. Rev. 103:53-71.

20. Caputo, A., Garner, R.S., Winkler, U., Hudig, D., and Bleackley, R.C. 1993. Activation of recombinant murine cytotoxic cell proteinase-1 requires deletion of an amino-terminal dipeptide. J. Biol. Chem. 268:17672-17675.

21. Pham, C.T.N., and Ley, T.J. 1999. Dipeptidyl peptidase I is required for the processing and activation of granzymes A and B in vivo. Proc. Natl. Acad. Sci. USA. 96:8627-8632.

22. Wolters, P.J., Pham, C.T.N., Muilenburg, D.J., Ley, T.J., and Caughey, G.H. 2001. Dipeptidyl peptidase I is essential for activation of mast cell chymases, but not tryptases, in mice. J. Biol. Chem. 276:18551-18556.

23. MacIvor, D.M., et al. 1999. Normal neutrophil function in cathepsin G-deficient mice. Blood. 94:4282-4293.

24. Edwards, J.C., Sedgwick, A.D., and Willoughby, D.A. 1981. The formation of a structure with the features of synovial lining by subcutaneous injection of air: an in vivo tissue culture system. J. Pathol. 134:147-156.

25. Liu, Z., et al. 2000. A critical role for neutrophil elastase in experimental bullous pemphigoid. J. Clin. Invest. 105:113-123.

26. Terato, K., et al. 1995. Collagen-induced arthritis in mice: synergistic effect of E. coli lipopolysaccharide bypasses epitope specificity in the induction of arthritis with monoclonal antibodies to type II collagen. Autoimmunity. 22:137-147.

27. Watson, W.C., Brown, P.S., Pitcock, J.A., and Townes, A.S. 1987. Passive transfer studies with type II collagen antibody in B10.D2/old and new line and $\mathrm{C} 57 \mathrm{Bl} / 6$ normal and beige (Chediak-Higashi) strains: evidence of important roles for $\mathrm{C} 5$ and multiple inflammatory cell types in the development of erosive arthritis. Arthritis Rheum. 30:460-465.

28. Choy, E.H.S., and Panayi, G.S. 2001. Cytokine pathways and joint inflammation in rheumatoid arthritis. N. Eng. J. Med. 344:907-916.

29. Cabrera, P.V., Blanco, G., Gravisaco, M.J., Alvarez, E., and Hajor, S. 2001. Zymosan modulates CD44 isoform expression in a murine model of inflammation resembling rheumatoid arthritis synovitis. J. Rheumatol. 28:943-949.

30. Salvesen, G., and Enghild, J.J. 1991. Zymogen activation specificity and genomic structures of human neutrophil elastase and cathepsin $\mathrm{G}$ reveal a new branch of the chymotrypsinogen superfamily of serine proteases. Biomed. Biochim. Acta. 50:665-671.

31. Gullberg, U., et al. 1999. Processing and targeting of granule proteins in human neutrophils. J. Immunol. Methods. 232:201-210.

32. Garwicz, D., Lindmark, A., Persson, A.-M., and Gullberg, U. 1998. On the role of the proform-conformation for processing and intracellular sorting of human cathepsin G. Blood. 92:1415-1422.

33. Stockley, R.A., Shaw, J., Afford, S.C., Morrison, H.M., and Burnett, D. 1990. Effect of alpha-1-proteinase inhibitor on neutrophil chemotaxis. Am. J. Respir. Cell Mol. Biol. 2:163-170.

34. Lomas, D.A., et al. 1995. The control of neutrophil chemotaxis by inhibition of cathepsin G and chymotrypsin. J. Biol. Chem. 270:23437-23443.

35. Coeshott, C., et al. 1999. Converting enzyme-independent release of tumor necrosis factor alpha and IL-1beta from a stimulated human monocytic cell line in the presence of activated neutrophils or purified proteinase 3. Proc. Natl. Acad. Sci. USA. 96:6261-6266.

36. Padrines, M., Wolf, M., Walz, A., and Baggiolini, M. 1994. Interleukin-8 processing by neutrophil elastase, cathepsin G and proteinase-3. FEBS Lett. 352:231-235.

37. Nufer, O., Corbett, M., and Walz, A. 1999. Amino-terminal processing of chemokine ENA-78 regulates biological activity. Biochemistry. 38:636-642.

38. Nakamura, H., Yoshimura, K., McElvaney, N.G., and Crystal, R.G. 1992. Neutrophil elastase in respiratory epithelial lining fluid of individuals with cystic fibrosis induces interleukin- 8 gene expression in a human bronchial epithelial cell line. J. Clin. Invest. 89:1478-1484.

39. Berger, S.P., et al. 1996. Proteinase-3, the major autoantigen of Wegener's granulomatosis, enhances IL-8 production by endothelial cells in vitro. J. Am. Soc. Nephrol. 7:694-701.

40. Blasi, F. 1993. Urokinase and urokinase receptor: a paracrine/autocrine system regulating cell migration and invasiveness. Bioessays. 15:105-111.

41. Brooks, P.C., et al. 1996. Localization of matrix metalloproteinase MMP-2 to the surface of invasive cells by interaction with integrin $\alpha v \beta 3$. Cell. 85:683-693.

42. Cai, T.-Q., and Wright, S.D. 1996. Human leukocyte elastase is an endogenous ligand for the integrin CR3 (CD11b/CD12, Mac-1, $\alpha \mathrm{M} \beta 2$ ) and modulates polymorphonuclear leukocyte adhesion. J. Exp. Med. 184:1213-1223.

43. Champagne, B., Tremblay, P., Cantin, A., and St. Pierre, Y. 1998. Proteolytic cleavage of ICAM-1 by human neutrophil elastase. J. Immunol. 161:6398-6405.

44. Levesque, J.-P., Takamatsu, Y., Nilsson, S.K., Haylock, D.N., and Simmons, P.J. 2001. Vascular cell adhesion molecule-1 (CD106) is cleaved by neutrophil proteases in the bone marrow following hematopoietic progenitor cell mobilization by granulocyte colony-stimulating factor. Blood. 98:1289-1297. 